\title{
Apertura de espacios de consumo higiénico y actuaciones policiales en zonas con fuerte tráfico de drogas. Evaluación mediante el recuento de las jeringas abandonadas en el espacio público
}

\author{
Safe injection rooms and police crackdowns in areas \\ with heavy drug dealing. Evaluation by counting \\ discarded syringes collected from the public space
}

\begin{abstract}
Carmen Vecino*; Joan R. Villalbí ***,***; Anna Guitart*,**; Albert Espelt*,**,***,****; Montserrat Bartroli*,**; Yolanda Castellano*,***; M Teresa Brugal*,**,***

* Agència de Salut Pública de Barcelona; ** Institut d’Investigació Biomèdica Sant Pau (IIB Sant Pau), Barcelona.

*** CIBER en Epidemiología y Salud Pública (CIBERESP); **** Departament de Psicobiologia i Metodologia de les Ciències de la Salut, Universitat Autònoma de Barcelona, Bellaterra.
\end{abstract}

\section{Resumen}

Se analiza la evolución de la inyección de drogas en espacios públicos usando como indicador el recuento del número de jeringuillas retiradas de la vía pública, evaluando además la influencia de las intervenciones sanitarias de reducción de daños y las policiales, mediante un estudio cuasi-experimental antes-después. Para cada intervención se contabilizan las jeringas recogidas mensualmente el semestre anterior y posterior tanto en el distrito implicado como en el conjunto de la ciudad, comparando mediante pruebas U y z la situación con un nivel de confianza del $95 \%$. La media mensual de jeringas recogidas disminuye de 13.132 en 2004 a 3.190 en 2012. Comparando los indicadores antes y después de la apertura de espacios de consumo higiénico y de acciones policiales se aprecia que la puesta en marcha de un espacio sanitario para el consumo supervisado de drogas en el casco antiguo se acompaña de una notable reducción de las jeringas abandonadas en la ciudad, y su volumen no varía en el distrito en que está ubicado. La posterior apertura de otro espacio de consumo no comporta cambios significativos en la media de jeringas abandonadas en la zona. Algunas acciones policiales en 200506 y 2011 tienen un impacto notable en los indicadores de los distritos en que se producen y del conjunto de la ciudad, mientras que otras no parecen tener el mismo efecto. Los programas de reducción de daños pueden tener un impacto favorable en el consumo de drogas inyectadas en el espacio público y el abandono de jeringas. Algunas intervenciones policiales parecen tener impacto, otras no, y otras un modesto efecto local y temporal.

\begin{abstract}
The evolution of drug injection in public places is analysed using as indicator the number of syringes collected from public spaces, evaluating as well the influence of public health harm reduction interventions and of police actions, with a before and after quasi experimental study. Monthly syringe counts on the semester before and after each intervention were compared both in the involved district and in the city as a whole, using the $\mathrm{U}$ and $\mathrm{z}$ tests with a $95 \%$ confidence level.

The average number of collected syringes drops from 13.132 in 2004 to 3.190 in 2012. Comparing indicators before and after health and police interventions, the opening of a facility with a supervised drug consumption room in the inner city was associated with a huge reduction in the number of abandoned syringes in the city, while its number did not rise in the district where the facility was located. The subsequent opening of another drug consumption room did not have a significant impact in collected syringes in the area. Some police interventions in 2005-2006 and 2011 had a significant impact in the indicators of the involved districts, while others did not. Harm reduction programs might have a favourable impact on drug injection in public spaces and related syringe presence. Some police interventions appear to have an impact while others do not or just have a modest local and temporary effect.
\end{abstract}

Key words: harm reduction, drug consumption room, street drugs, program evaluation, police forces, syringes. 


\section{Introducción}

A lgunos usuarios de drogas por vía inyectada consumen y abandonan el material de inyección en el espacio público (de la Fuente, Bravo, Toro, Brugal y Barrio, 2005). Esto comporta un cierto riesgo de transmisión de enfermedades infecciosas si se produce un pinchazo accidental, además de generar miedo y rechazo social al asociarse en el imaginario colectivo la jeringa abandonada al usuario de drogas (Babor et al, 2010; García Algar y Vall, 1997). Para mitigar este problema se han desarrollado estrategias que incluyen los programas de intercambio de jeringas (PIJ), los buzones de eliminación higiénica, y los programas de recogida selectiva. Los servicios de salud pública han organizado sistemas de recogida y eliminación higiénica de estos residuos, para disminuir los riesgos de punción accidental y la alarma social; en Barcelona estos programas se iniciaron tentativamente en el casco antiguo (Bechich, Martínez, Llorella, Rodés, de Andrés y Delás, 2001), y se ampliaron luego a otras zonas. En 2004 el programa de recogida abarcaba tres distritos, y actualmente cubre siete de los diez distritos de la ciudad.

En el verano del 2004 la ampliación del puerto de Barcelona comportó el derribo del poblado de Can Tunis, un enclave de viviendas sociales alejado del casco urbano que concentraba buena parte del tráfico de drogas de la ciudad. Éste se desplazó entonces a otras zonas de la ciudad y de la región metropolitana, destacando dentro del término municipal de Barcelona el tráfico en el casco antiguo (distrito de Ciutat Vella), en otras zonas cercanas al puerto (distrito de Sants-Montjuïc), y en diversos polígonos de viviendas sociales. Al retornar al casco urbano, el tráfico se acompañó de una mayor visibilidad del consumo, y de la reaparición de jeringas abandonadas en espacios públicos. Por este motivo se pusieron en marcha diversas intervenciones con el objetivo de paliar esta problemática. Desde el punto de vista sanitario se ampliaron los servicios de atención a las drogodependencias y se adoptaron y reforzaron programas de reducción de daños, destacando los espacios de consumo supervisado de drogas; desde el punto de vista de control de la oferta de drogas, las fuerzas policiales desarrollaron diversas actuaciones.

Los objetivos del presente estudio son estudiar la evolución de la inyección de drogas en espacios públicos a partir del recuento del número de jeringuillas retirado de la vía pública como indicador, evaluando la influencia de las intervenciones sanitarias de reducción de daños y las policiales, con datos de un período de ocho años de julio de 2004 a junio de 2012 .

\section{Método}

\section{Diseño y análisis}

Se trata de un estudio cuasi-experimental antes-después (López, Marí-dell'Olmo, Pérez-Giménez y Nebot, 2011). Se analiza la influencia de la apertura de espacios de consumo higiénico como intervenciones sanitarias de reducción de daños y de las actuaciones policiales sobre el tráfico en el número de jeringas abandonadas en cada zona. Para cada intervención mayor (sanitaria o policial) se estiman para el semestre anterior y posterior la media mensual de jeringas recogidas en el distrito implicado y en el conjunto de la ciudad, así como la proporción que las jeringas recogidas en el distrito representan dentro del conjunto de jeringas recogidas en la ciudad. Se considera como semestre posterior al que se inicia tras la culminación de la intervención cuando ésta se desarrolla a lo largo de un período, y semestre anterior a los seis meses previos. Se comparan las distribuciones antes y después de la intervención con la prueba $\mathrm{U}$ de Mann-Whitney, y se realiza la prueba z para la comparación de proporciones, en ambos casos con un nivel de confianza del $95 \%$. Además, se presenta el número de jeringas recogidas por territorio desde julio de 2004, año en que se formaliza esta actividad hasta junio de 2012.

\section{Recogida territorial de datos}

La ciudad de Barcelona está organizada administrativamente en diez distritos (figura 1), cada uno de los cuales incluye diversos barrios, y el sistema de información sobre drogas de la ciudad ha analizado sistemáticamente los datos por unidades territoriales (Brugal, Domingo-Salvany, Maguire, Caylà, Villalbí 1999). La información sobre jeringuillas recogidas por territorio se obtiene de los registros de retirada de jeringuillas del espacio público. El programa de recogida de jeringas es una actividad coordinada desde los servicios de salud pública. En ella participan diversos actores: servicios de limpieza municipales, Instituto Municipal de Parques y Jardines, educadores comunitarios de salud pública, y pacientes que asisten a talleres de reinserción. Todos los agentes disponen de contenedores higiénicos y siguen un procedimiento normalizado para la recogida. Mensualmente, los

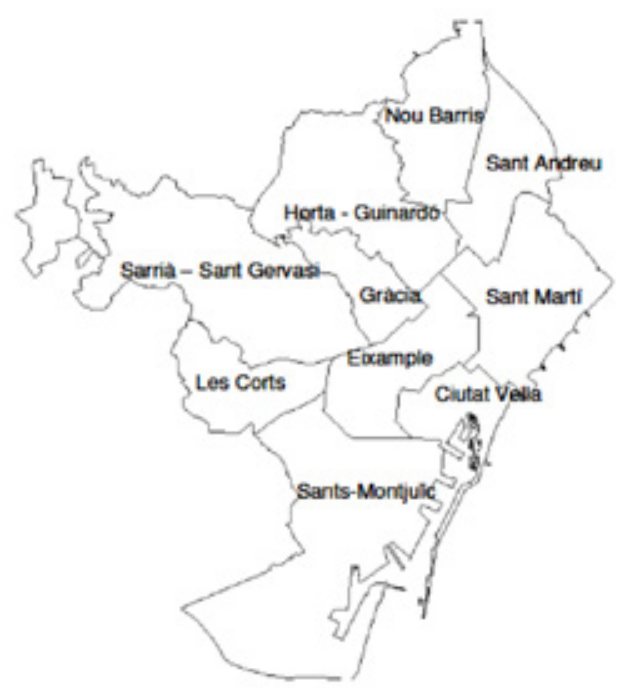

Figura 1. Mapa con la división administrativa de la ciudad de Barcelona en distritos 
servicios de salud pública reciben los contenedores para su eliminación de acuerdo a lo prescrito como residuos sanitarios (Departament de Presidència, 1999), junto con el recuento de jeringas recogidas por cada agente, que se compilan en un fichero. En 2004 el programa abarcaba tres distritos: Ciutat Vella, Sants-Montjuïc, y Nou Barris. En marzo de 2007 se amplía al distrito de Sant Andreu, y en marzo de 2008 al distrito de Sant Martí. Los distritos del Eixample y Horta también disponen del servicio, pero como entre ambos distritos se recogen menos de dos jeringas a la semana de media, no se incluyen en este trabajo.

\section{Intervenciones y cronología}

Las intervenciones sanitarias en el ámbito de las drogodependencias en la ciudad se impulsan y coordinan desde la Agència de Salut Pública de Barcelona, que tiene encomendado velar por la ejecución del Plan de Acción sobre Drogas, tanto en lo que respecta a los servicios que gestiona directamente como a los realizados por otros actores (Brugal, Espelt y Ricart, 2009). Las intervenciones policiales se conocen en el marco de los espacios de coordinación existentes entre los servicios de salud pública y otros servicios relevantes ante la problemática de la droga. Ambos tipos de intervención se incorporan a los registros del Servicio de Prevención y Atención a las Drogodependencias para ser incluidas en la evaluación de los Planes de Acción sobre Drogas que se realizan desde 1989, lo que proporciona una cronología detallada.

En 2004 se refuerzan los espacios de consumo higiénico inyectado ya operativos previamente en el distrito de Sants-Montjuïc (dos plazas en una unidad móvil) y se mantiene el existente en el mismo Raval de Ciutat Vella (dos plazas en el SAPS). En diciembre de 2004, se refuerzan los programas de reducción de daños en Ciutat Vella, incrementando el intercambio de jeringas y programas con usuarios y abriendo un nuevo espacio de acogida y consumo higiénico, la Sala Baluard (seis plazas). En 2005 se abre el CAS Vall d'Hebron en el distrito de Horta, con otro pequeño espacio de consumo higiénico (una plaza). En diciembre de 2011 se acondiciona otro pequeño espacio de consumo supervisado en el CAS Forum del distrito de Sant Martí (una plaza), que empieza a acoger actividad el dos de enero. Además hay otros cambios en los dispositivos de atención ambulatoria a las adicciones. En 2010-11 se pone en marcha el CAS Forum, se integra la atención a drogas en el centro de salud mental de Gracia, se inicia tratamiento ambulatorio de baja exigencia en la Sala Baluard (que se convierte en un CAS), y se traslada a nuevas dependencias uno de los centros de atención situados en Ciutat Vella, que se desplaza a otro barrio sin salir de este distrito.

A lo largo de estos años se llevan a cabo distintas intervenciones policiales dirigidas a reducir el tráfico de drogas. Destacan entre ellas seis operaciones mayores: de noviembre de 2005 a febrero del 2006 en el distrito de Nou Barris (Porta); en enero y febrero de 2006 en Ciutat Vella (Raval); en junio de 2008 en el distrito de Sants-Montjuïc (Zona Franca); de agosto a octubre de 2008 en Ciutat Vella (Raval); en febrero de 2009 en Ciutat Vella (Gòtic); en diciembre de 2011 en Sant Andreu (Trinitat Vella).

\section{Resultados}

La media mensual de jeringas recogidas disminuye a lo largo de estos ocho años, de 13.132 en 2004 a 3.190 en 2012 pese a que el sistema de información cubría inicialmente tres distritos y ahora abarca cinco. El distrito con mayor número de jeringas abandonadas en el espacio público es Ciutat Vella que incluye el casco antiguo, seguido por Sants-Montjuïc que incluye otras zonas vecinas a las instalaciones portuarias. En Nou Barris se reducen hasta casi desaparecer, y en Sant Andreu se registra un incremento temporal que luego disminuye. Hay una fuerte caída en 2005 y 2006, y posteriormente el indicador se mantiene estable entre 2007 y 2010 (con oscilaciones entre distritos), y se detecta una nueva disminución en 2011. El número de jeringas recogidas en el espacio público en 2012 es el 24,3\% del que era en 2004. En el distrito de Ciutat Vella, que es donde se recogían más jeringas de los espacios públicos y donde hay más servicios de reducción de daños, en 2012 es sólo el 21,2\% del inicial (figura 2).

En la tabla 1 se comparan los indicadores antes y después de cada una de las intervenciones sanitarias y policiales. Como puede verse, la puesta en marcha a finales de 2004 del espacio de consumo supervisado en Ciutat Vella se acompaña de una notable reducción de las jeringas abandonadas en la ciudad, mientras que su volumen no varía en el distrito en que está ubicado. La actividad policial de 200506, con acciones en dos distritos distintos, tiene un impacto notable en todos los indicadores de estas zonas y del conjun-

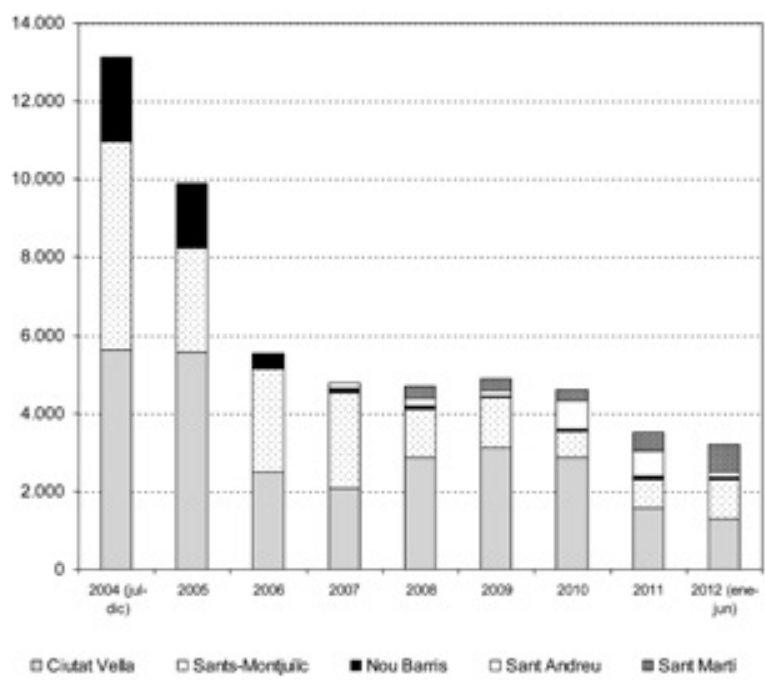

Figura 2. Número medio de jeringas mensuales recogidas de la vía pública, por distrito y año. Barcelona, 2004-12 
Tabla 1

Jeringas recogidas del espacio público antes y después de diversas intervenciones. Barcelona, 2004-2012

\begin{tabular}{|c|c|c|c|c|}
\hline Indicadores de jeringas recogidas según suceso & $\begin{array}{l}\text { Semestre } \\
\text { anterior }\end{array}$ & $\begin{array}{l}\text { Semestre } \\
\text { posterior }\end{array}$ & Razón & $\mathbf{p}$ \\
\hline \multicolumn{5}{|l|}{ Apertura sala consumo en Ciutat Vella, diciembre 2004} \\
\hline Media mensual de jeringas recogidas en el distrito & 5.631 & 5.924 & 1,05 & NS \\
\hline Media mensual de jeringas recogidas en el conjunto de la ciudad & 13.132 & 10.449 & 0,80 & $<0,01$ \\
\hline$\%$ del total de jeringas recogidas en el distrito & $42,8 \%$ & $56,7 \%$ & 1,32 & $<0,00001$ \\
\hline Media mensual de jeringas recogidas en el distrito de Nou Barris & 1.286 & 343 & 0,27 & $<0,01$ \\
\hline Media mensual de jeringas recogidas en el distrito de Ciutat Vella & 4.432 & 2.468 & 0,56 & $<0,01$ \\
\hline Media mensual de jeringas recogidas en el conjunto de la ciudad & 7.822 & 5.432 & 0,69 & NS \\
\hline$\%$ del total de jeringas recogidas en el distrito de Nou Barris & $16,4 \%$ & $6,31 \%$ & 0,38 & $<0,00001$ \\
\hline \multicolumn{5}{|l|}{ Operaciones policiales en Sants-Montjuïc (Zona Franca), junio de 2008} \\
\hline Media mensual de jeringas recogidas en el distrito & 1.327 & 1.146 & 0,86 & NS \\
\hline Media mensual de jeringas recogidas en el conjunto de la ciudad & 3.773 & 5.624 & 1,49 & $<0,01$ \\
\hline$\%$ del total de jeringas recogidas en el distrito & $32,2 \%$ & $20,5 \%$ & 0,64 & $<0,00001$ \\
\hline \multicolumn{5}{|l|}{ Operaciones policiales en Ciutat Vella (Raval), agosto - octubre 2008} \\
\hline Media mensual de jeringas recogidas en el distrito & 3.550 & 2.815 & 0,79 & NS \\
\hline Media mensual de jeringas recogidas en el conjunto de la ciudad & 5.508 & 4.374 & 0,79 & NS \\
\hline$\%$ del total de jeringas recogidas en el distrito & $64,5 \%$ & $64,7 \%$ & 1,00 & NS \\
\hline \multicolumn{5}{|l|}{ Operaciones policiales en Ciutat Vella (Gòtic), febrero 2009} \\
\hline Media mensual de jeringas recogidas en el distrito & 3.313 & 3.122 & 0,94 & NS \\
\hline Media mensual de jeringas recogidas en el conjunto de la ciudad & 5.004 & 5.159 & 1,03 & NS \\
\hline \multicolumn{5}{|l|}{$\begin{array}{l}\text { Operaciones policiales en Sant Andreu (Trinitat Vella) y apertura espacio } \\
\text { consumo en CAS situado en Sant Martí, diciembre } 2011\end{array}$} \\
\hline Media mensual de jeringas recogidas en el distrito de Sant Andreu & 611 & 129 & 0,21 & $<0,00001$ \\
\hline Media mensual de jeringas recogidas en el distrito de Sant Martí & 368 & 692 & 1,88 & NS \\
\hline Media mensual de jeringas recogidas en el conjunto de la ciudad & 3.536 & 3.190 & 0,90 & $<0,00001$ \\
\hline$\%$ del total de jeringas recogidas en el distrito de Sant Andreu & $17,3 \%$ & $4,0 \%$ & 0,23 & $<0,00001$ \\
\hline$\%$ del total de jeringas recogidas en el distrito de Sant Martí & $10,4 \%$ & $21,7 \%$ & 2,09 & $<0,00001$ \\
\hline
\end{tabular}

to de la ciudad, que además se mantiene en años sucesivos. La intervención policial de mediados de diciembre de 2011 en el barrio de Trinitat Vella de Sant Andreu parece tener un fuerte impacto; la apertura casi simultánea de un espacio de consumo supervisado en el CAS Forum en diciembre de 2011 (que inició su actividad el 2 de enero) comporta un incremento del volumen de jeringas recogidas en la zona, que no alcanza la significación estadística. Tras estas dos intervenciones casi simultáneas, policial y sanitaria, se da un declive en el volumen de jeringas recogidas en el conjunto de la ciudad. Las intervenciones policiales de los años 2008 y 2009 no parecen tener un efecto mayor: aunque se acompañen de reducciones en la problemática del distrito que no alcanzan la significación estadística, no impactan en los indicadores del conjunto de la ciudad (que en un caso incluso se incrementan tras la intervención). Sin embargo, en dos casos el efecto local no es despreciable, pues tras la intervención policial disminuye el peso relativo de la problemática del distrito implicado en el conjunto de la ciudad.

\section{Discusión}

Los principales resultados del estudio son que la puesta en marcha de un espacio de consumo supervisado en una zona con fuerte tráfico y consumo de drogas se acompaña de una notable reducción de las jeringas abandonadas en la ciudad, sin que su magnitud varíe en el distrito en que está ubicado el recurso. La posterior apertura de un pequeño espacio de consumo en otra zona carece de impactos estadísticamente significativos. Además, se aprecia que algunas intervenciones policiales tienen un impacto positivo en la reducción de jeringuillas abandonadas tanto en las zonas de 
actuación como en el resto de la ciudad, mientras que otras no muestran ninguna diferencia.

La distribución temporal y territorial de las jeringas recogidas del espacio público proporciona indicadores útiles para la gestión y evaluación. Refleja los cambios en tráfico y consumo, y puede ser una herramienta de vigilancia si se usa de forma sistemática. Algunas intervenciones policiales parecen tener impacto duradero, mientras que otras carecen de él o tienen sólo un impacto local, desplazando temporalmente la problemática a otro territorio sin modificar las cifras globales del problema, algo descrito también en otros medios (Wood et al., 2004a). Pero hay que destacar que la puesta en marcha de un espacio de consumo supervisado cerca de una zona con fuerte tráfico y consumo de drogas se acompañó de una reducción de los indicadores de problemática en la ciudad, sin comportar un incremento local de los mismos: esto es muy relevante para el futuro, para poder planificar acciones basadas en datos.

Las jeringas recogidas marcan las zonas de tráfico de drogas, y su distribución es un indicador intuitivo (que en nuestra experiencia es apreciado por los responsables políticos y utilizado en espacios de participación). Este indicador proporciona información territorial y temporal con cierta estabilidad, aunque existan elementos que pueden actuar como factor de confusión (como la identificación y limpieza de un espacio de consumo previamente desconocido, que comporta un pico puntual de jeringas en la zona implicada). El circuito de obtención de datos es más rápido que otros componentes del sistema de información. Pese a ser un indicador indirecto, proporciona información para monitorizar el consumo visible de drogas inyectadas en el espacio público con un coste bajo. Tan sólo hemos encontrado referencias a su uso como indicador en las ciudades canadienses de Vancouver y Montreal (Montigny, Vernez-Moudon, Leigh y Young, 2010; Wood et al., 2004b).

A lo largo de este periodo el número de inyectores en la ciudad se ha mantenido estable de acuerdo con los indicadores de mortalidad, inicios de tratamiento y atención en urgencias que monitoriza el Sistema de Información de Drogas de Barcelona (Espelt, Queralt, Graugés, García, Vecino y Brugal, 2012). Por un lado se han incorporado nuevos usuarios, especialmente procedentes de países del Centro y Este de Europa (González, Cebrián, Nadal, Sala, Vall-llosera y Delás, 2003), pero por otro lado los usuarios locales que se inyectan han disminuido (Barrio et al., 2012). La venta de jeringas en las farmacias no está sujeta a limitaciones en España, y no se identifican cambios en la accesibilidad a jeringas de los usuarios inyectores, aunque puedan darse modificaciones locales en una u otra zona.

Hay que tener en cuenta algunas limitaciones de este trabajo. En primer lugar, el estudio se circunscribe a la ciudad de Barcelona y el área metropolitana que la incluye es un continuo urbano, en el que los límites administrativos no afectan a la dinámica del consumo de drogas. Las acciones que se realizan en la ciudad tienen efectos también fuera de ella, aunque éstos no se pueden reflejar en este estudio que se basa en el sistema de información sobre drogas de Barcelona. Así, la apertura del espacio de consumo en el CAS Forum se produce en un barrio colindante con una zona de fuerte tráfico pero que está fuera de los límites de la ciudad (el barrio de la Mina del municipio de Sant Adrià del Besós): aunque la apertura del espacio en el CAS Forum pueda haber tenido un fuerte impacto en la Mina, el estudio no puede apreciarlo por no disponer de datos de otras ciudades. En segundo lugar, el principal factor impulsor de los programas de recogida de jeringas es la alarma social expresada mediante las quejas de los residentes, y al tener esta una naturaleza subjetiva podría plantear algún sesgo entre territorios: un mismo fenómeno puede ser percibido de forma diversa según barrios. De todos modos, creemos que la naturaleza compacta de la ciudad y su elevada densidad lo hacen improbable. Finalmente, al utilizar el distrito como unidad de análisis perdemos la perspectiva de lo que sucede en unidades territoriales más pequeñas, como los barrios que lo componen. En este sentido, es posible que algunas intervenciones tengan efectos en ámbitos pequeños, desplazando el tráfico y el consumo de drogas de una zona a otra dentro de un mismo distrito, que no tienen reflejo cuantitativo en los datos agregados por distrito. Pese a estas limitaciones, que quizás puedan abordarse en futuros estudios, creemos que el trabajo aporta datos de interés. Además, confirma empíricamente que la implantación de servicios de reducción de daños no incrementa la problemática del consumo en el espacio público ni el volumen de jeringas abandonadas, sino que se acompaña de una reducción notable de su magnitud, como también apuntaba un trabajo previo en Vancouver (Canadá) (Wood, Tyndall, Montaner y Kerr, 2006). Esto es relevante, pues los programas de reducción de daños son intervenciones muy eficaces para la prevención de enfermedades infectocontagiosas (Bravo et al., 2009; de la Fuente et al., 2006), pero el temor a que planteen molestias locales o favorezcan la extensión de las adicciones y la ausencia de datos empíricos publicados ha frenado su desarrollo: nuestros resultados sugieren que estos temores son en buena parte infundados, y esta es una información importante para la definición de políticas públicas.

La diversidad de efecto observada de las distintas intervenciones policiales es explicable: se ha documentado la falta de impacto de las acciones policiales que sólo alcanzan a los eslabones finales de la cadena de tráfico, reemplazados con relativa facilidad. Sin embargo, cuando la investigación policial permite desarticular al núcleo de una red de traficantes de nivel medio el impacto puede ser importante, y éste podría ser el caso de las intervenciones de finales de 2005 e inicios de 2006, así como la de diciembre de 2011 (Babor et al., 2010). De todas maneras hay que tener en cuenta que la elasticidad de la demanda de sustancias por parte de los usuarios ante mayores costes económicos o de 
transacción es relativamente modesta, por lo que las acciones policiales suelen desplazar el tráfico (que arrastra el consumo) a otras zonas, incluso fuera del ámbito municipal y por tanto fuera de nuestra capacidad de registrarlas. La experiencia de Vancouver documenta como una operación policial de gran intensidad en la zona con mayor tráfico y consumo de drogas generó un desplazamiento temporal del tráfico y consumo a las zonas adyacentes sin alterar la frecuencia de consumo ni el precio de la droga; hubo una disminución de las jeringas recogidas en la zona, con un incremento concomitante en otras zonas (Wood et al., 2004 a) .

\section{Reconocimientos}

Los autores desean expresar su agradecimiento a los diversos actores implicados en la recogida de jeringas: Àmbit Prevenció, ABD, SAPS Cruz Roja, Instituto Municipal de Parques y Jardines de Barcelona, Barcelona pel Medi Ambient, y todas las personas que contribuyen a la recogida de jeringas de la vía pública. Este trabajo ha sido parcialmente financiado con la ayuda de la Red de Investigación Cooperativa (RETIC) de Trastornos Adictivos (RTA), Expediente RD06/0001/1018.

\section{Conflicto de intereses.}

Los autores declaran no estar sujetos a conflicto de intereses.

\section{Referencias}

Babor T., Caulkins J., Edwards G., Fisher B., Foxcroft D., Humpheys K., ... Strang, J. (2010). Drug policy and the public good. Oxford: Oxford University Press.

Barrio, G., Bravo, M.J., Brugal, M.T., Díez, M., Regidor, E., Belza, M.J. y de la Fuente, L. (2012). Harm reduction interventions for drug injectors or heroin users in Spain: expanding coverage as the storm abates. Addiction, 107, 1111-1122. doi: 10.1111/j.1360-0443.2011.03759.x.

Bechich, S., Martínez, M., Llorella, G., Rodés, A., De Andrés, M. y Delás, J. (2001). RECOJE: recogida comunitaria de jeringas por usuarios de drogas por vía parenteral como complemento a los programas de intercambio. Gaceta Sanitaria, 15, 353-355.

Bravo, M.J., Royuela, L., de la Fuente, L., Brugal, M.T., Barrio, G. y Domingo-Salvany, A. (2009). Use of supervised injection facilities and injection risk behaviours among young drug injectors. Addiction, 104, 614-619. doi: 10.1111/j.1360-0443.2008.02474.x

Brugal, M.T., Domingo-Salvany, A., Maguire, A., Caylà, J.A., Villalbí, J.R. y Hartnoll, R. (1993). A small area analysis estimating the prevalence of addiction to opioids in Barcelona. Journal of Epidemiology and Community Health, 53, 488-494.
Brugal, M.T., Espelt, A. y Guitart, A. (2009). Pla d'acció sobre drogues de Barcelona 2009-12. Barcelona: Agència de Salut Pública de Barcelona. Recuperado de http://www.aspb. cat/quefem/docs/Pla_drogues_2009_12.pdf.

Departament de Presidència. Decret 27/1999, de 9 de febrer, de la gestió dels residus sanitaris. Diari Oficial de la Generalitat de Catalunya 2828, 16.2.99.

De la Fuente, L., Brugal, M.T., Ballesta, R., Bravo, M.J., Barrio, G., Domingo, A.,.. y grupo del proyecto ITINERE. (2005). Metodología del estudio de cohortes del proyecto ITINERE sobre consumidores de heroína en tres ciudades españolas y características básicas. Revista Española de Salud Pública, 79, 475-491.

De la Fuente, L., Bravo, M.J., Toro, C., Brugal, M.T. y Barrio, G. (2006). Injecting and HIV prevalence among young heroin users in three Spanish cities and their association with the delayed implementation of harm reduction programmes. Journal of Epidemiology and Community Health, 60, 537-542.

Espelt, A., Queralt, A., Graugés, D., García, V., Vecino, C. y Brugal, M.T. (2012). Sistema d'Informació de Drogodependències de Barcelona. Actualització dels indicadors de drogues corresponent al 3er i 4rt Trimestre de 2011. Barcelona: Agència de Salut Pública de Barcelona. Recuperado de http://www.aspb.cat/quefem/docs/sidb/default.htm.

García-Algar, O. y Vall, O. (1997). Hepatitis B virus infection from a needle stick. Pediatric Infectious Disease Journal, 16, 1099.

González, M., Cebrián, S., Nadal, C., Sala, L., Vall-llosera, A. y Delás, J. (2003). Ciudadanos del este de Europa consumidores de drogas en Barcelona. Gaceta Sanitaria 17, 256-268.

López, M.J., Marí-Dell'Olmo, M., Pérez-Giménez, A. y Nebot, M. (2011). Diseños evaluativos en salud pública: aspectos metodológicos. Gaceta Sanitaria, 25 (Mon 1), 9-16.

Montigny, L., Vernez-Moudon, A., Leigh, B. y Young, K. (2010). Assessing a drop box programme: A spatial analysis of discarded needles. International Journal of Drug Policy, 21, 208-214. doi: 10.1016/j.drugpo.2009.07.003

Wood, E., Spittal, P., Small, W., Kerr, T., Li, K., Hogg, R.S., ... Schechter, M.T. (2004 a). Displacement of Canada's largest public illicit drug market in response to a police crackdown. Canadian Medical Association Journal, 170, 1551-1556. doi: 10.1503/cmaj.1031928

Wood, E., Kerr, T., Small, W., Li, K., Marsh, D.C., Montaner, J.S.G. y Tyndall, M.W. (2004 b). Changes in public order after the opening of a medically supervised safer injecting facility for illicit injection drug users. Canadian Medical Association Journal 171, 731-734.

Wood, E., Tyndall, M.W., Montaner, J.S. y Kerr, T. (2006). Summary of findings from the evaluation of a pilot medically supervised safer injecting facility. Canadian Medical Association Journal, 175, 1399-1404. 